\title{
Transcriptomic Analysis Predicts Survival and Sensitivity to Anticancer Drugs of Patients with a Pancreatic Adenocarcinoma
}

Pauline Duconseil, ${ }^{*}$ Marine Gilabert, ${ }^{*}$ Odile Gayet, ${ }^{*}$ Celine Loncle, ${ }^{*}$ Vincent Moutardier, ${ }^{* \dagger}$ Olivier Turrini, ${ }^{*}$ Ezequiel Calvo, ${ }^{\S}$ Jacques Ewald, ${ }^{\ddagger}$ Marc Giovannini, ${ }^{\ddagger}$ Mohamed Gasmi, " Erwan Bories, ${ }^{\ddagger}$ Marc Barthet, " Mehdi Ouaissi, ${ }^{\natural}$ Anthony Goncalves, Flora Poizat, ${ }^{\ddagger}$ Jean Luc Raoul, ${ }^{\ddagger}$ Veronique Secq, ${ }^{* \dagger}$ Stephane Garcia, ${ }^{* \dagger}$ Patrice Viens,,${ }^{\ddagger}$ Juan Iovanna, ${ }^{*}$ and Nelson Dusetti*

From the Cancer Research Center of Marseille (CRCM),* INSERM U1068, CNRS UMR 7258, Aix-Marseille University and Paoli-Calmettes Institute, Scientific and Technological Park of Luminy, Marseille, France; the Departments of Surgery ${ }^{\dagger}$ and Gastroenterology, ${ }^{\top}$ Hôpital Nord, Marseille, France; the Paoli-Calmettes Institute, ${ }^{\ddagger}$ Marseille, France; the Genomic Center, ${ }^{\S}$ CHUL Research Centre, Quebec City, Quebec, Canada; and the Department of Surgery, La Timone Hospital, Marseille, France

Accepted for publication November 25, 2014.

Address correspondence to Nelson Dusetti, Ph.D., or Juan Iovanna, M.D., Ph.D., INSERM U1068, $163 \mathrm{Av}$ de Luminy, Batiment TPR2, 13288 Marseille, France. E-mail:nelson.dusetti@ inserm.fr or juan.iovanna@ inserm.fr.

\begin{abstract}
A major impediment to the effective treatment of patients with pancreatic ductal adenocarcinoma (PDAC) is the molecular heterogeneity of this disease, which is reflected in an equally diverse pattern of clinical outcome and in responses to therapies. We developed an efficient strategy in which PDAC samples from 17 consecutive patients were collected by endoscopic ultrasound-guided fine-needle aspiration or surgery and were preserved as breathing tumors by xenografting and as a primary culture of epithelial cells. Transcriptomic analysis was performed from breathing tumors by an Affymetrix approach. We observed significant heterogeneity in the RNA expression profile of tumors. However, the bioinformatic analysis of these data was able to discriminate between patients with long- and shortterm survival corresponding to patients with moderately or poorly differentiated PDAC tumors, respectively. Primary culture of cells allowed us to analyze their relative sensitivity to anticancer drugs in vitro using a chemogram, similar to the antibiogram for microorganisms, establishing an individual profile of drug sensitivity. As expected, the response was patient dependent. We also found that transcriptomic analysis predicts the sensitivity of cells to the five anticancer drugs most frequently used to treat patients with PDAC. In conclusion, using this approach, we found that transcriptomic analysis could predict the sensitivity to anticancer drugs and the clinical outcome of patients with PDAC. (Am J Pathol 2015, 185: 1022-1032; http://dx.doi.org/10.1016/j.ajpath.2014.11.029)
\end{abstract}

Pancreatic ductal adenocarcinoma (PDAC) is one of the most lethal human malignancies and a major health problem. PDAC causes approximately 230,000 deaths per year worldwide. ${ }^{1}$ Despite considerable research efforts in the past 20 years, conventional treatment approaches, including surgery, radiation, chemotherapy, and a combination of these, have limited impact. The prognosis is dismal, with only $20 \%$ of patients alive 1 year after diagnosis. ${ }^{1}$ Given this scenario, the search for new treatments to counter PDAC progression and thereby increase patient life expectancy has been given high priority. Particularly, future therapeutic agents are expected to be molecularly targeted to specifically affect PDAC cells by targeting their weaknesses while leaving normal tissues undamaged. Recently, studies that reached phase 3 clinical trials in unselected PDAC populations tested bevacizumab, erlotinib, and axitinib, which are molecularly targeted agents, combined with gemcitabine. These trials did not show robust survival benefits, probably because they were essayed on unselected PDAC populations that were highly heterogeneous. ${ }^{2-4}$ In fact, a major impediment to the effective treatment of PDAC is the

Supported by La Ligue Contre le Cancer, Institut National du Cancer, Canceropole PACA, SIRIC PACA-OUEST, and INSERM (all to J.I.).

P.D., M.Gil., O.G. and C.L. contributed equally to this work.

Disclosures: None declared. 
molecular heterogeneity of the disease, reflected in diverse clinical response patterns to therapy. Hence, the starting point of this work was the heterogeneous evolution observed in patients with PDAC, with survival of 2 to 3 months to 5 years after diagnosis and with a strong difference in susceptibility to classical and novel drugs. This may be explained by the fact that each PDAC has a combination of several modifications in intracellular pathways that may result in variable susceptibility to drugs, metastasis development, and, hence, survival. ${ }^{5-7}$ The treatments proposed to the patients do not take into account this heterogeneity. Yet, the drugs received by patients with PDAC are chosen according to their general performance status and the stage of their disease. No study of the tumor can predict its responsiveness to the treatment or give a prognosis to the disease progression. For example, the objective response rate was $31.6 \%$ in multidrug FOLFIRINOX-treated patients and only $9.4 \%$ in patients treated with gemcitabine, showing that nearly $70 \%$ and $90 \%$ of the patients, respectively, did not respond. ${ }^{8}$ Another possibility that explains the resistance to systemic therapies is that PDAC tumors are hypovascularized and very rich in stroma, which can represent $15 \%$ to $90 \%$ of the tumor mass, resulting in a barrier to drug delivery to the transformed cells. ${ }^{9}$

We hypothesized that the variable sensitivity observed in patients with PDAC is mainly due to the intrinsic molecular characteristics of the cancer cells. To characterize this heterogeneity, we obtained 17 novel human PDAC-derived xenografts and established their corresponding primary cultures. We then characterized the 17 PDAC-derived xenografts at the transcriptomic level. The analysis of these data showed significant heterogeneity. However, the bioinformatic study of transcriptome discriminated that patients with long- and short-term survival corresponded to moderately and poorly differentiated histopathologic phenotypes, respectively. We treated the PDAC-derived primary cell cultures with different doses ( 0 to $1000 \mu \mathrm{mol} / \mathrm{L}$ ) of five anticancer drugs and found, as expected, that responses were patient dependent. Using this approach, we also found that the transcriptomic analysis was able to predict the sensitivity of cancer cells to the five anticancer drugs most frequently used in PDAC patients.

\section{Materials and Methods}

\section{PDAC Samples}

Patients were included in this project under the Paoli Calmettes Institute clinical trial number 2011-A01439-32. Three expert clinical centers collaborated on this project after receiving ethics review board approval. Patient informed consent forms were collected and registered in a central database. The tumor tissue used for xenograft development was deemed excess to that required for the patient's diagnosis. Two types of samples were obtained: endoscopic ultrasound-guided fine-needle aspiration (EUS-FNA) biopsy samples from patients with unresectable tumors and tumor tissue samples from patients undergoing surgery. All the samples were anonymized, and postsurgical anatomopathology reports were provided for specimens from each patient. Histopathologic evaluation was performed on 5- $\mu \mathrm{m}$ hematoxylin and eosin-stained sections of patient tumors and xenografts and examined under a light microscope. These sections were compared with the original human tumor when available.

It is obvious that studies on cancer cells, analyzed on whole surgical specimens, introduce a high background in systemic analysis. Therefore, we optimized the conditions of primary culture for PDAC cells obtained by surgery and by EUS-FNA, a technique systematically used for diagnosis in our hospitals. Each sample obtained from EUS-FNA was mixed with $100 \mu \mathrm{L}$ of Matrigel (BD Biosciences, Franklin Lakes, NJ) and was injected in the upper right flank of a nude mouse (Swiss Nude Mouse Crl: NU(lco)-Foxn1nu; Charles River Laboratories, Wilmington, MA). Each sample derived from surgery resection was fragmented, mixed with $100 \mu \mathrm{L}$ of Matrigel, and implanted with a trocar (10 gauge; Innovative Research of America, Sarasota, FL) in the subcutaneous right upper flank of an anesthetized and disinfected mouse. When the tumors reached $1 \mathrm{~cm}^{3}$, the mice were sacrificed, and the tumors were removed. Xenografts that failed to develop within 6 months were discontinued. The study on animals was approved by the Animal Facility and Experimental Platform (Scientific and Technological Park of Luminy, Marseille, France).

\section{Xenografted Tumor Processing and Cell Culture}

Xenografts obtained from mice were split into several small pieces and used for cell culture, histologic analyses, reimplantation into new mice, and freezing. The fragments designated for cell culture were processed in a biosafety chamber: after fine mincing, they were treated with collagenase type $\mathrm{V}$ (ref C9263; Sigma-Aldrich, St. Louis, MO) and trypsin/EDTA (ref 25200-056; Gibco, Life Technologies, Grand Island, NY) and were suspended in Dulbecco's modified Eagle's medium supplemented with $1 \% \mathrm{w} / \mathrm{w}$ penicillin/streptomycin (Gibco, Life Technologies) and 10\% fetal bovine serum (Lonza Inc., Walkersville, MD). After centrifugation, cells were resuspended in serum-free ductal media adapted from Schreiber et $\mathrm{ll}^{10}$ at $37^{\circ} \mathrm{C}$ in a $5 \% \mathrm{CO}_{2}$ incubator. Amplified cells were stored in liquid nitrogen. Cells were weaned from antibiotics for $\geq 48$ hours before testing.

\section{Gene Expression Microarrays}

For the preparation of RNA samples, xenografted tumors were removed from mice at passage 2 and immediately drawn in a cold 4-mol/L guanidinium thiocyanate solution, followed by protein denaturation and RNA extraction according to Chirgwin's protocol. ${ }^{11}$ Total RNA $(1.0 \mu \mathrm{g})$ was reverse transcribed for hybridization to the human oligonucleotide array Human Gene 2.0 (GeneChip; Affymetrix Inc., Santa Clara, CA) as described previously. ${ }^{12}$ Arrays were 


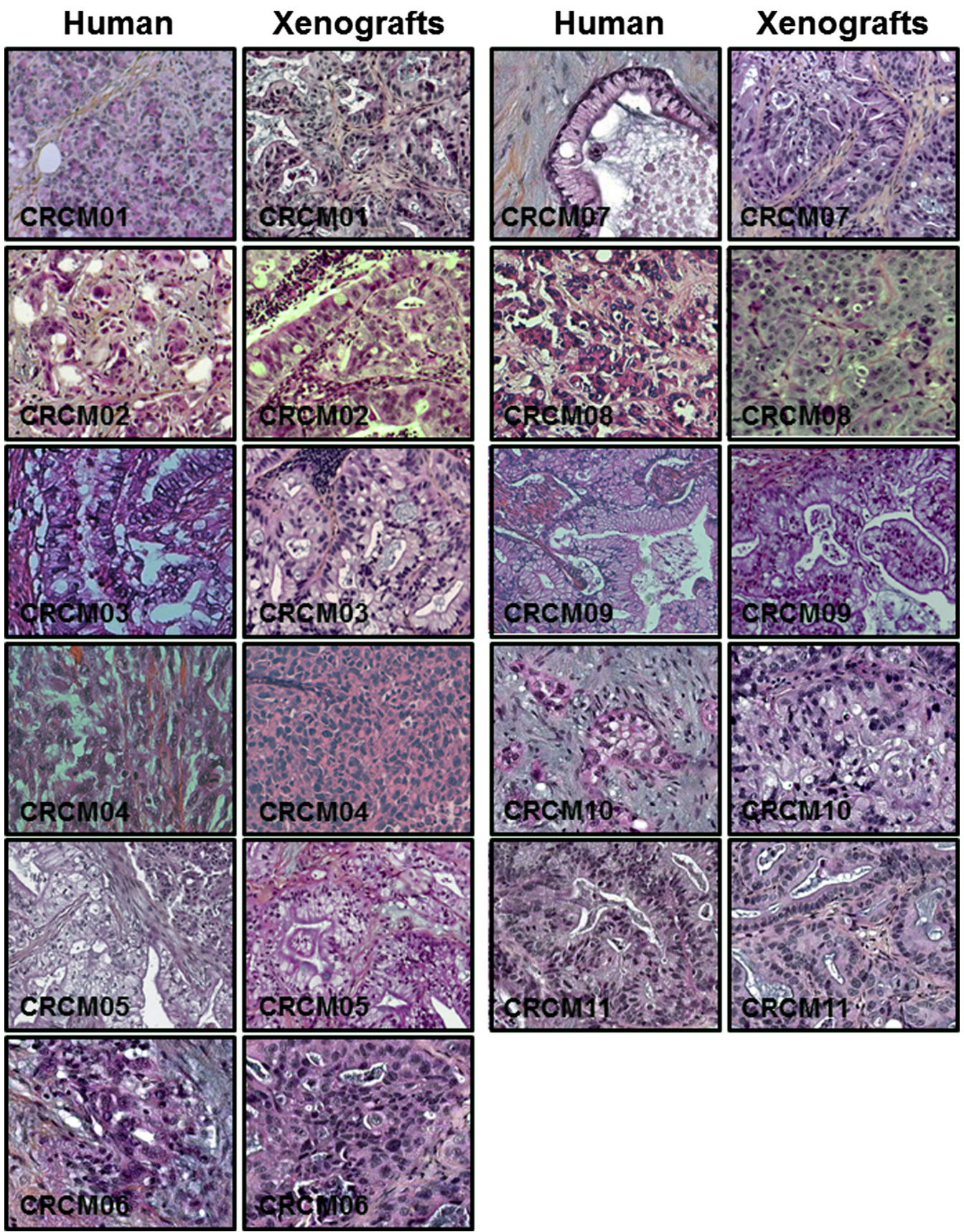

Figure 1 Hematoxylin and eosin staining of pancreatic ductal adenocarcinoma (PDAC) samples from xenografted tumors obtained by surgery and the original PDAC samples obtained by pancreatectomy.

processed using the Affymetrix GeneChip fluidic station 450 (protocol EukGE-WS2v5_450) and were scanned using a GeneChip scanner 3000 G7 (Affymetrix Inc.). GeneChip operating software version 1.4 (Affymetrix Inc.) was used to obtain chip images and for quality control. Background subtraction and normalization of probe set intensities were performed using the method of robust multiarray analysis. ${ }^{12}$ Principal component analysis was performed using Genomics Suite software version 6.6 (Partek Inc., St. Louis, MO). Gene set enrichment analysis was performed using the Broad Institute platform, and statistical significance (false discovery rate) was set at 0.25 . Microarray analysis was performed by the CHU de Québec Research Center Gene Expression Platform (Quebec City, Quebec, Canada). All array data are available on the Gene Expression Omnibus (http://www.ncbi.nlm.nih.gov/ geo; Accession number GSE55513).

\section{Chemograms}

Cells were screened for chemosensitivity to five clinically used drugs in patients with PDAC: gemcitabine (Eli Lilly \& Co., Indianapolis, IN), 5-fluorouracil (5FU; Teva Pharmaceutical 
Industries, Petah Tikva, Israel), oxaliplatin (Hospira, Lake Forest, IL), docetaxel (Sanofi, Bridgewater, NJ), and irinotecan active metabolite named 7-ethyl 10-hydroxycamptothecin or SN-38 (H-0165-10 MG; Sigma-Aldrich). These cells were treated for 72 hours with increasing concentrations of chemotherapeutic drugs ranging from 0 to $1000 \mu \mathrm{mol} / \mathrm{L}$ and from 0 to $100 \mu \mathrm{mol} / \mathrm{L}$ for $\mathrm{SN}-38$. Five thousand cells were plated per well in 96-well plates in serum free defined media. Twenty-four hours later, the media were supplemented with increasing concentrations of drugs and were incubated for an additional 72 hours. Each experiment was performed in triplicate and repeated at least three times. Cell viability was estimated after addition of the PrestoBlue cell viability reagent (Life Technologies) for 3 hours following the protocol provided by the supplier. Cell viability was measured on days 0 and 3 to calculate the replication rate of the cells.

\section{Clinical Outcome}

The clinical outcomes of the 17 patients were recorded in a prospective database, and data such as age, sex, medical history, histopathologic stage of differentiation, treatments received (type and duration of chemotherapy), response to chemotherapy, date of progression, metastasis occurrence and localization, and date of death were analyzed. Progressionfree and overall survival were then calculated. Short-term survival was arbitrarily defined as a survival time $\leq 8$ months, and long-term survival was defined as a survival time $>8$ months.

\section{Results}

\section{Correlation between Transcriptome and Clinical Outcome}

Eleven primary tumors obtained from surgery (Figure 1) and six biopsy samples from EUS-FNA (Figure 2) were implanted subcutaneously into mice and preserved as xenografts by reimplantation. The histopathologic and clinical characteristics of the patients are displayed in Supplemental Table S1. Histologically, all the specimens were PDACs, which were moderately to poorly differentiated. Growth rates to reach a tumor volume of $1 \mathrm{~cm}^{3}$ ranged from 2 to 6 months. Histopathologic comparisons between patients' primary tumors and xenografts were performed. Hematoxylin and eosin-stained tissue sections showed that xenografts retained the original morphologic features of the human primary tumor (Figure 1). The relative state of differentiation was also retained on passaging until at least six times (data not shown). As is typical of PDAC, a high degree of stroma density was observed in the primary tumors but was progressively lost in xenografts.

Total RNA was obtained from 17 xenografts, and the transcriptome was performed using Affymetrix microchips. The first approach to grouping patients by their expression

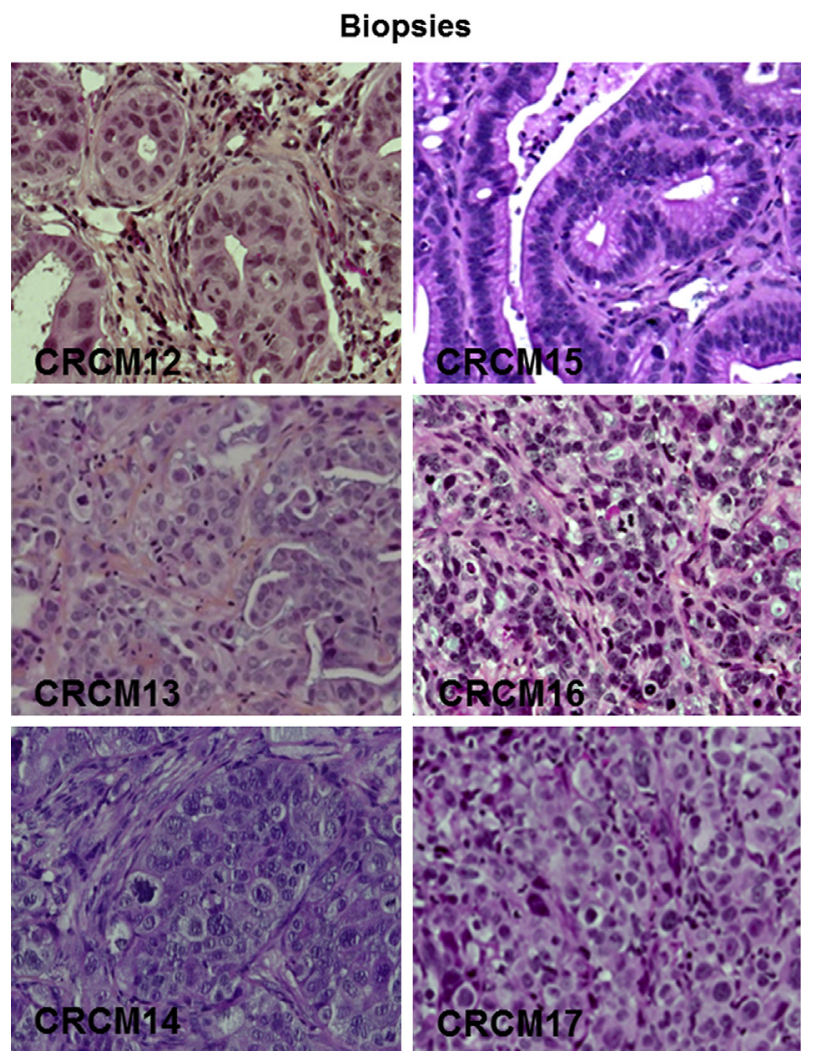

Figure 2 Hematoxylin and eosin staining of pancreatic ductal adenocarcinoma samples from xenografted tumors obtained by endoscopic ultrasound-guided fine-needle aspiration biopsy.

profile used a principal component analysis. We found that the transcriptome from two patient-derived xenografts, CRCM04 and CRCM17, highly detached from the main group of patients (Figure 3A). We analyzed their clinical data and found that both corresponded to two particularly short-term survival patients with poorly differentiated PDAC (Figures 1, 2, and 3, A and B). We then explored the presence of other short-term survival patients with poorly differentiated PDAC in our collection and found that three additional patient-derived xenografts, CRCM16, CRCM08, and CRCM14, corresponded to patients with short survival times of $<8$ months (Figure 3B) with poorly differentiated tumors (Figures 1 and 2).We found that their transcriptome result was relatively close to the CRCM04 and CRCM17 ones, indicating a relatively similar expression profile in short-term survival patients with poorly differentiated tumors (Figure 3A). Of the 11 samples obtained from surgery, only 2 corresponded to short-term survivors, whereas of the six samples obtained by EUS-FNA, three corresponded to short-term survivors. In fact, it is not a surprise because samples obtained by EUS-FNA corresponded to patients not suitable for surgery as first-line treatment and, therefore, to tumors that have a worse prognosis.

We next performed a heat map analysis to identify all genes differentially expressed between short- and longterm survival patients. A total of 505 genes appeared as 
overexpressed and 439 genes as underexpressed in shortterm compared with long-term survivors (Figure 3C and Supplemental Table S2). A Gene Ontology analysis, with a fold change $>1.5$ and a false discovery rate $>0.001$, on differential expression showed significant gene enrichment in biological processes associated with cancer, such as cell cycle, mitosis, response to cellular stress, DNA metabolism, chromosome organization, and cellular metabolism (Supplemental Figures S1-S6). Most of these genes were overexpressed in short-term survival with poorly differentiated PDAC, suggesting that these pathways were preferentially activated.

\section{Chemogram}

Seventeen PDAC primary cultures were obtained from human PDAC xenografts. Cells were characterized to assess their epithelial origin by immunofluorescence with CK19 antibody, Kras mutation was identified by PCR followed by DNA sequencing, and their transformed status was confirmed by their capacity to produce subcutaneous tumors after injection of $10^{7}$ cells in nude mice. All these primary cell cultures are strongly stained with the antiCK19 antibody; they are all mutated in codons 12,13 , or 61 and produce tumors in mice within the 8 weeks that follow the injection (data not shown). Fibroblast contamination was estimated by immunofluorescence using the anti-S100A4 antibody. No more than 5\% fibroblast contamination was observed. Cells in primary culture were microscopically analyzed, presenting a high degree of heterogeneity between different patients (Figure 4A). Cell cultures were considered stable after 10 passages and were used during the next 10 passages at maximum to prevent clonal selection.

To study the sensitivity to chemotherapeutical drugs, the cells were submitted to increasing concentrations (from 0.001 to $1000 \mu \mathrm{mol} / \mathrm{L}$ ) of a set of anticancer drugs and their sensitivity measured to obtain a dose-response curve characterizing each patient. Using this approach we were able to estimate their relative chemosensitivity. To establish a chemoresistance score for all the drugs, we selected the concentration of each drug in which the difference between patients was the largest. Based on these data, we classified patients from more resistant to more sensitive (Table 1). Each patient-derived cell line showed its own chemogram (Figure 5 and Table 1). CRCM04, CRCM12, CRCM15, CRCM16, and CRCM09 were the five most sensitive patient-derived cell lines to gemcitabine; CRCM03, CRCM16, CRCM04, CRCM12, and CRCM05 were the most sensitive to SN-38 (the active irinotecan metabolite); CRCM04, CRCM10, CRCM06, CRCM14, and CRCM13 were the most sensitive to
A

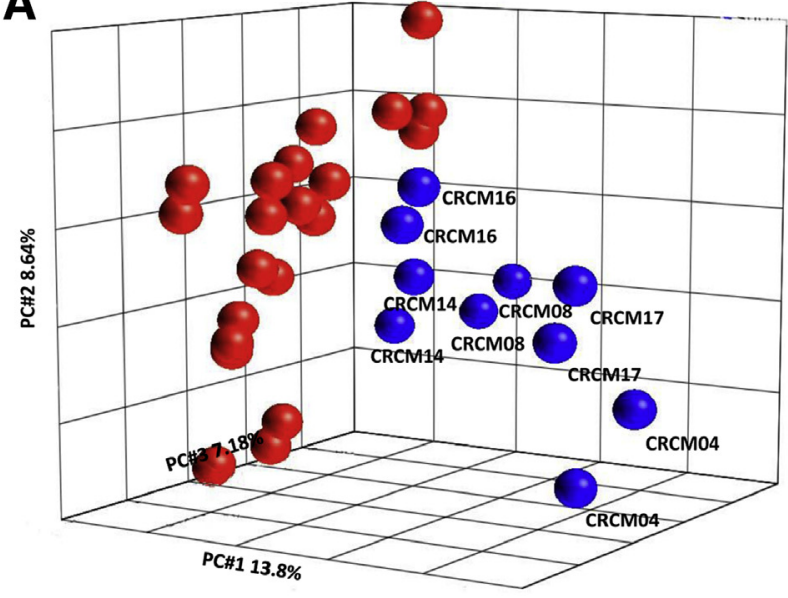

B

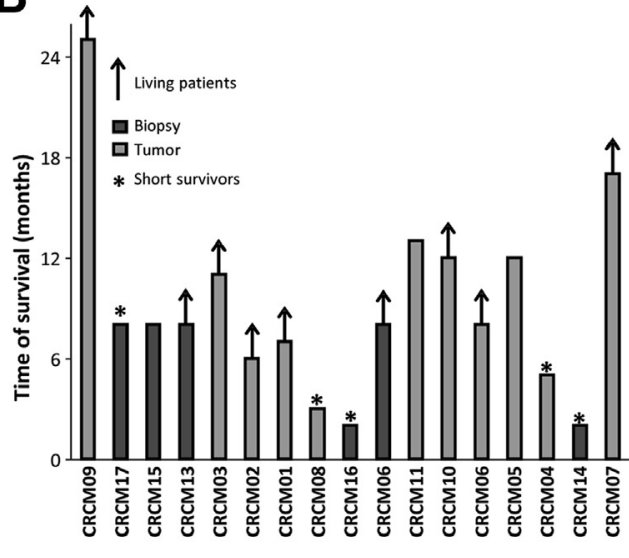

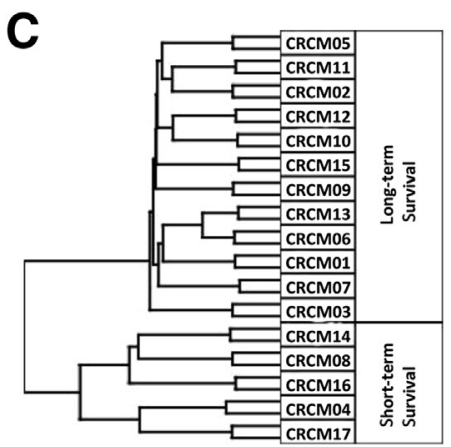

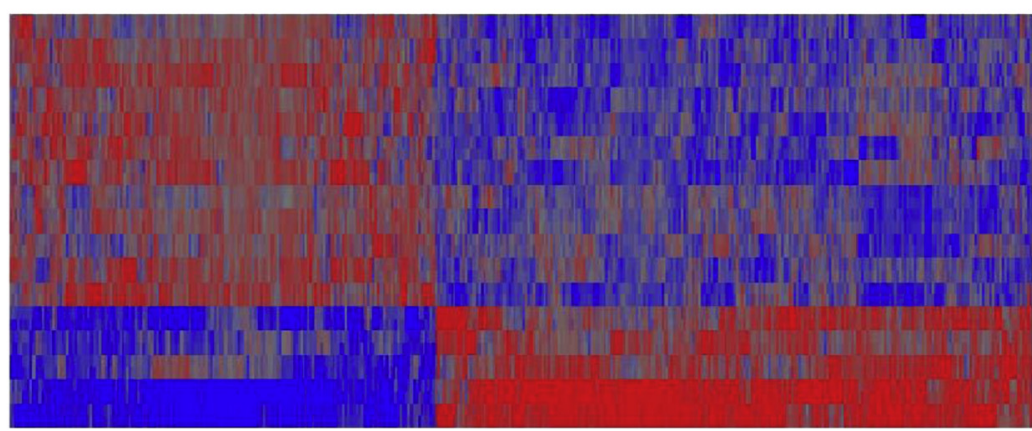

Figure 3 RNA expression analysis of pancreatic ductal adenocarcinoma. A: Principal component analysis obtained from the human oligonucleotide array Human Gene 2.0 Affymetrix data. Each patient is represented in duplicate because data were obtained from two independents experiments. Red plots represent long-term survival patients; blue plots, short-term survival patients. B: Histogram of the survival times of patients included in this study. C: Heat map shows the RNA expression profile of patients with long- and short-term survival. 


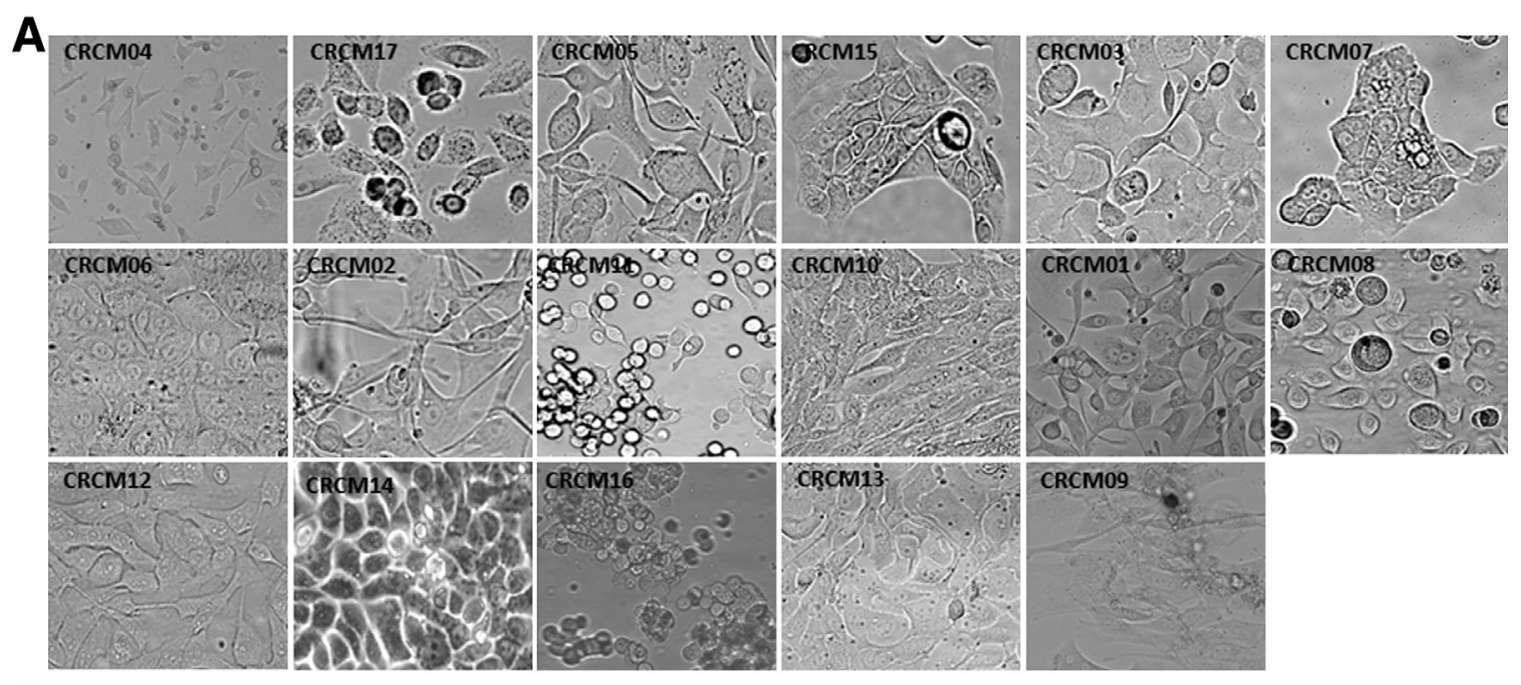

B

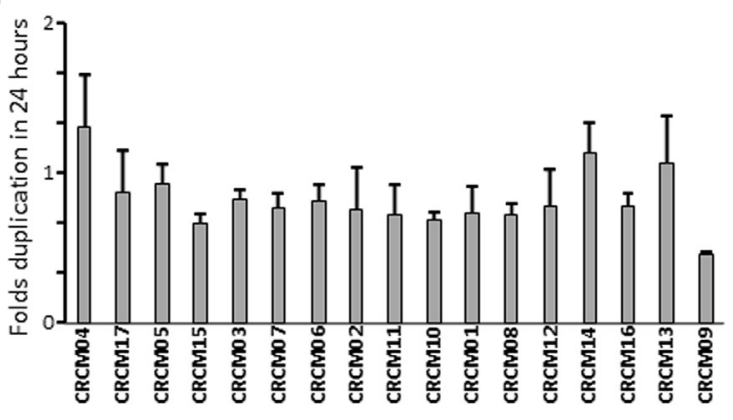

Figure 4 Primary cell cultures of pancreatic ductal adenocarcinoma samples. A: Contrast phase microscopy pictures representing the 17 primary cell cultures. B: Histogram representing the duplication folds in 24 hours of each cell line. Data are given as means \pm SD.

5FU; CRCM14, CRCM04, CRCM07, CRCM16, and CRCM06 were the most sensitive to oxaliplatin; and CRCM04, CRCM08, CRCM15, CRCM14, and CRCM16 were the most sensitive to docetaxel (Table 1). We found that the five most resistant cell lines were CRCM14, CRCM13, CRCM06, CRCM17, and CRCM11 to gemcitabine; CRCM09, CRCM17,

Table 1 Resistance Score by Cell Line

\begin{tabular}{llllll}
\hline Score & Gemcitabine & Docetaxel & 5FU & 0xaliplatin & SN-38 \\
\hline 1 & CRCM04 & CRCM04 & CRCM04 & CRCM14 & CRCM03 \\
2 & CRCM12 & CRCM08 & CRCM10 & CRCM04 & CRCM16 \\
3 & CRCM15 & CRCM15 & CRCM06 & CRCM07 & CRCM04 \\
4 & CRCM16 & CRCM14 & CRCM14 & CRCM16 & CRCM12 \\
5 & CRCM09 & CRCM16 & CRCM13 & CRCM06 & CRCM05 \\
6 & CRCM10 & CRCM06 & CRCM07 & CRCM13 & CRCM06 \\
7 & CRCM08 & CRCM05 & CRCM05 & CRCM10 & CRCM15 \\
8 & CRCM03 & CRCM10 & CRCM09 & CRCM15 & CRCM13 \\
9 & CRCM05 & CRCM12 & CRCM03 & CRCM17 & CRCM07 \\
10 & CRCM07 & CRCM03 & CRCM15 & CRCM05 & CRCM14 \\
11 & CRCM01 & CRCM07 & CRCM17 & CRCM09 & CRCM01 \\
12 & CRCM02 & CRCM13 & CRCM16 & CRCM02 & CRCM08 \\
13 & CRCM11 & CRCM02 & CRCM12 & CRCM03 & CRCM11 \\
14 & CRCM17 & CRCM11 & CRCM01 & CRCM11 & CRCM10 \\
15 & CRCM06 & CRCM17 & CRCM11 & CRCM01 & CRCM02 \\
16 & CRCM13 & CRCM01 & CRCM08 & CRCM12 & CRCM17 \\
17 & CRCM14 & CRCM09 & CRCM02 & CRCM08 & CRCM09 \\
\hline
\end{tabular}

5FU, 5-fluorouracil.
CRCM02, CRCM10, and CRCM11 to SN-38; CRCM02, CRCM08, CRCM11, CRCM01, and CRCM12 to 5FU; CRCM08, CRCM12, CRCM01, CRCM11, and CRCM03 to oxaliplatin; and CRCM09, CRCM01, CRCM17, CRCM11, and CRCM02 to docetaxel (Table 1). Altogether, these results suggest that each PDAC-derived primary culture line has a particular profile of response. In other words, sensitivity or resistance to one drug does not predict sensitivity or resistance to another drug.

After 72 hours of incubation, some drugs were unable to kill all the cells even at very high concentrations, such as $1000 \mu \mathrm{mol} / \mathrm{L}$ for gemcitabine, oxaliplatin, docetaxel, or $5 \mathrm{FU}$ or $100 \mu \mathrm{mol} / \mathrm{L}$ for $\mathrm{SN}-38$ (Figure 5). For example, 20\% to $50 \%$ of cells were resistant to $1000 \mu \mathrm{mol} / \mathrm{L}$ of gemcitabine; $5 \%$ to $30 \%$ remained alive when treated with $100 \mu \mathrm{mol} / \mathrm{L}$ of $\mathrm{SN}-38 ; 10 \%$ to $70 \%$ when treated with $1000 \mu \mathrm{mol} / \mathrm{L} 5 \mathrm{FU}$; and $0 \%$ to $25 \%$ for $1000 \mu \mathrm{mol} / \mathrm{L}$ oxaliplatin. A particular behavior was found when cells were treated with docetaxel; whereas doses of some drugs as low as $0.001 \mu \mathrm{mol} / \mathrm{L}$ were able to kill $90 \%$ to $20 \%$, depending on the primary culture, increasing the doses to $3.9 \mu \mathrm{mol} / \mathrm{L}$ resulted in almost no change in their effect. However, almost all the cells were killed by $62.5 \mu \mathrm{mol} / \mathrm{L}$. These results suggest that there is more than one cellular population in the tumor, each with different sensitivity, including complete insensitivity to drugs, which is in agreement with the intratumoral heterogeneity of PDAC. For this reason, we believe that the 

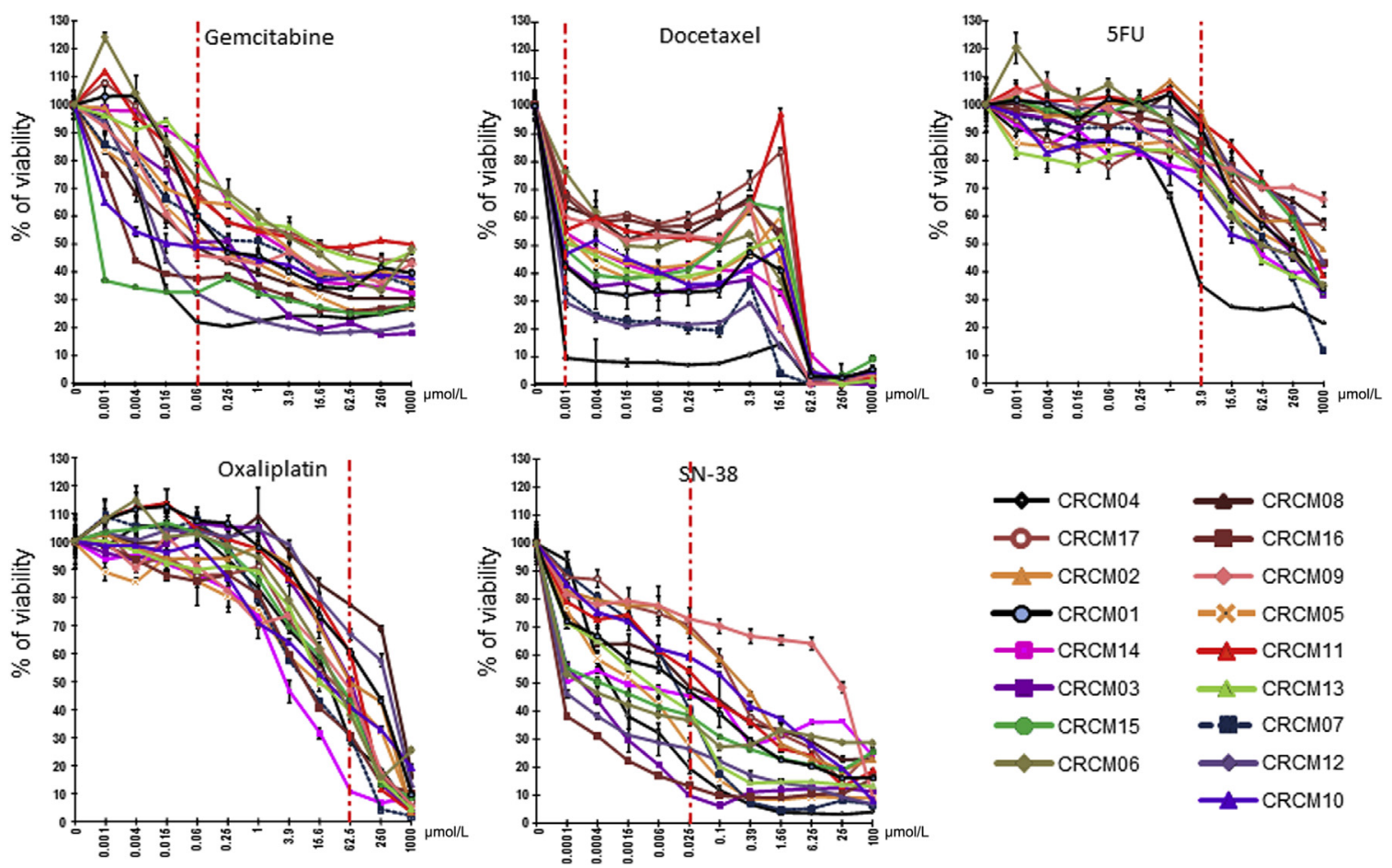

Figure 5 Chemograms. Pancreatic ductal adenocarcinoma-derived cells were treated with increasing concentrations of gemcitabine, docetaxel, 5 fluorouracil (5FU), oxaliplatin, and the active metabolite of irinotecan known as SN-38, and the survival cells were measured after 72 hours of treatment. A sensitivity profile was obtained for each drug. The point indicated with a red dotted line was used to establish the score used in Table 1.

inhibitory concentration of $50 \%$ value is not useful to characterize drug sensitivity for primary cultured cells.

\section{Correlation between Transcriptome and Drug Resistance in PDAC}

To study whether there is a correlation between drug response and transcriptome, we arbitrarily selected the four most sensitive and the four most resistant primary cultures and performed a heat map analysis of the transcriptome. Sets of genes were identified as specifically overexpressed or underexpressed in resistant and sensitive cells (Figure 6). These modulations in the expression profiles allowed us to identify 671 genes associated with gemcitabine, 1107 with oxaliplatin, 308 with 5FU, 149 with docetaxel, and only 46 with SN-38 (Figure 6A and Supplemental Tables S3-S7, respectively). Ninety-eight genes were common between oxaliplatin and 5FU, which is in agreement with their close mechanism of action. On the contrary, there were a small number of common genes associated with sensitivity and resistance to the other drugs. Few genes were observed to be common between different drugs (Figure 6B), suggesting that the phenotype of the sensitivity or resistance is specific for each drug.

Last, another important point is that genes associated with sensitivity or resistance to the drugs were not common with genes associated with short- or long-term survival (Figure 7), indicating that survival and drug sensitivity are regulated by independent mechanisms.

\section{Discussion}

We developed an efficient strategy in which PDAC tumors from 17 consecutive patients were collected, their cells maintained as primary cultures, and surgical specimens and EUS-FNA biopsy samples as breathing tumors by xenografting in immunosuppressed mice. For all the patients, clinical follow-up was conducted. Primary culture of cells allowed analysis of their relative sensitivity to several anticancer drugs in vitro, obtaining an individual chemogram. We studied the RNA expression profile using a microarray approach on breathing tumors. We used xenografts as a source of RNA rather than cultured cells because their morphologic features and microenvironment, which both have a determinant effect on RNA expression, ${ }^{13}$ were closer to the original human PDAC. These data were used to detect a possible correlation with the clinical outcome of each patient and with their drug responsiveness.

Methodologically, note that we were able to obtain PDAC samples from EUS-FNA and from surgically resected tumors. Biopsy samples obtained from EUS-FNA containing only some hundreds of tumor cells were able to reconstitute a tumor in mice with morphologic features very close to those of the primary tumors (Figure 1). This was systematically observed when comparing xenografts removed from mice with the histologic slide of the same resected tumor after the patient's surgery. This original strategy opens up new possibilities of clinical interest. We can imagine studying clinical outcome or the presence of a 
A
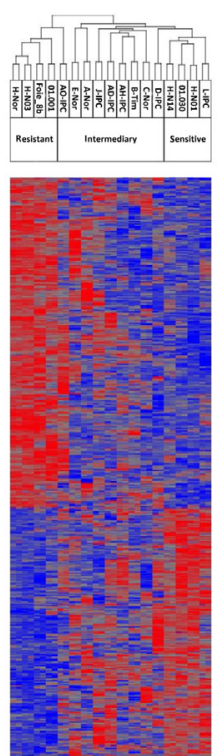

Gemcitabine
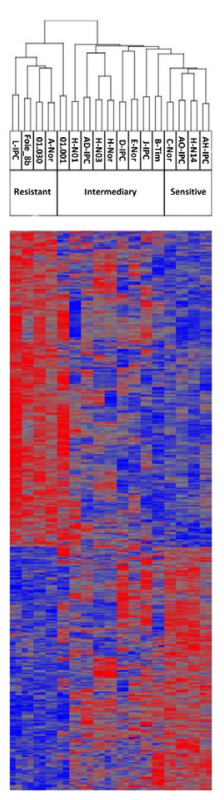

Oxaliplatin
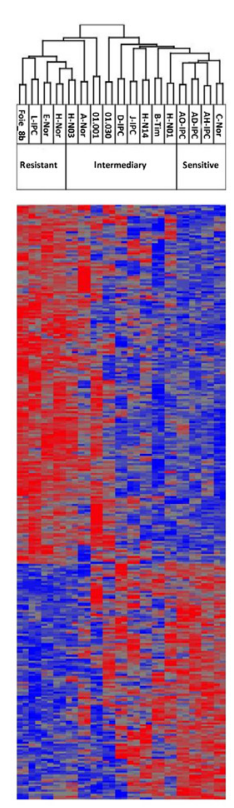

$5 \mathrm{FU}$
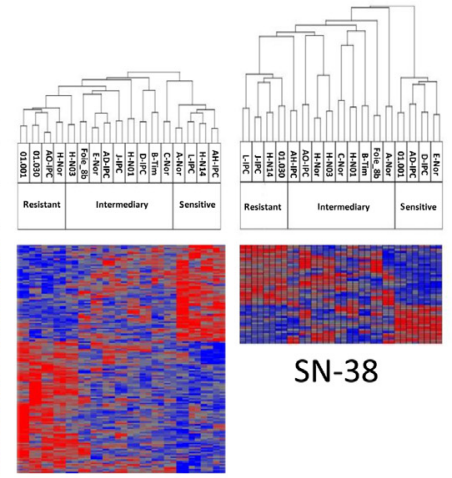

SN-38

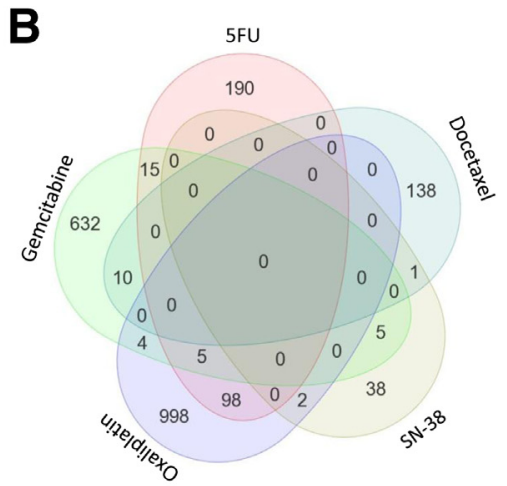

Docetaxel

Figure 6 RNA expression and drug sensitivity. A: Heat maps showing the RNA expression profiles of pancreatic ductal adenocarcinoma-derived cells with resistance or sensitivity to the treatments with gemcitabine, docetaxel, 5-fluorouracil (5FU), oxaliplatin, and SN-38. B: A Venn diagram showing the limited (except for 5FU and oxaliplatin) number of genes (in common) associated with resistance or sensitivity to the treatments.

drug-responsive transcriptomic signature in every PDAC patient to personalize treatment. Indeed, not only could the $15 \%$ to $20 \%$ of operated patients benefit from individualized treatments but virtually all patients with a PDAC because they undergo EUS-FNA biopsy before starting chemotherapy or radiotherapy.

Analysis of gene profiling of whole PDAC tumors has largely been reported. ${ }^{14-24}$ However, the low reproducibility of data discouraged us from continuing in this direction. The reasons for the failure of this approach are probably associated with inappropriate strategies, inefficient tools, and the poor quality of the samples, and they can also be inherent to the pathologic disorder itself because PDAC tumors can contain $15 \%$ to $90 \%$ of stroma associated with variable areas of inflammation and necrosis. An efficient approach using laser capture of the transformed epithelial cells followed by their pangenomic expression analysis was more recently reported. ${ }^{25}$ This approach demonstrated that there is a significant correlation between the expression profile and the patient outcome, including the responsiveness to some treatments. One of the limitations was that analysis was performed on only surgically resected tumors, which correspond to a minority of patients with a relatively better prognosis because of earlier detection. The other limitation was that tissue samples were obtained from formalin-fixed, paraffin-embedded tissue specimens collected between 1993 and 2006, and the quality of the obtained RNA could be deficient. ${ }^{26}$ Using the EUS-FNA approach, all the patients can be included and RNA extracted from fresh tissue, providing a better quality of RNA samples for analysis.

One strength of this study is the predictive capacity of the RNA profile obtained to separate at least two groups of

patients: those with short-term survival ( $\leq 8$ months) and those with long-term survival ( $>8$ months). Analysis of clinical data from short- and long-term survival patients showed that the first group had poorly differentiated tumors, whereas long-term survival patients presented with middleor well-differentiated tumors. As expected, this observation matched with the morphologic features observed in xenografted tumors (Figure 1). Therefore, these data are not surprising because tumors with low-grade differentiation are expected to be associated with short-term survival compared with high- or middle-grade differentiation. ${ }^{27-30}$ This correlation between tumor differentiation and prognosis was already described for pancreatic neuroendocrine tumors, ${ }^{31}$ and its pertinence in PDAC was recently documented by Wasif et $\mathrm{al}^{32}$ who described a correlation between tumor differentiation and patient survival time as well as a value to predict the response to treatment. In fact, tumor differentiation or grade is increasingly used as an independent prognostic factor of survival, it seems with as much impact as prognostic tumor size or lymph node metastatic invasion. ${ }^{33}$ Although the grade of differentiation can be estimated by the pathologist after a pancreatectomy, this is possible in only approximately $15 \%$ of patients. However, using a set of molecular markers identified in this study, differentiation could be estimated using only a few cells obtained by EUS-FNA. In fact, based on the present results, its seems evident that using the expression of some of these molecular indicators on small PDAC samples as obtained by EUS-FNA would help in predicting the behavior of the PDAC and, therefore, conditioning the therapeutic strategy. Although this application sounds very exciting, it needs to be validated in an independent cohort of patients. 


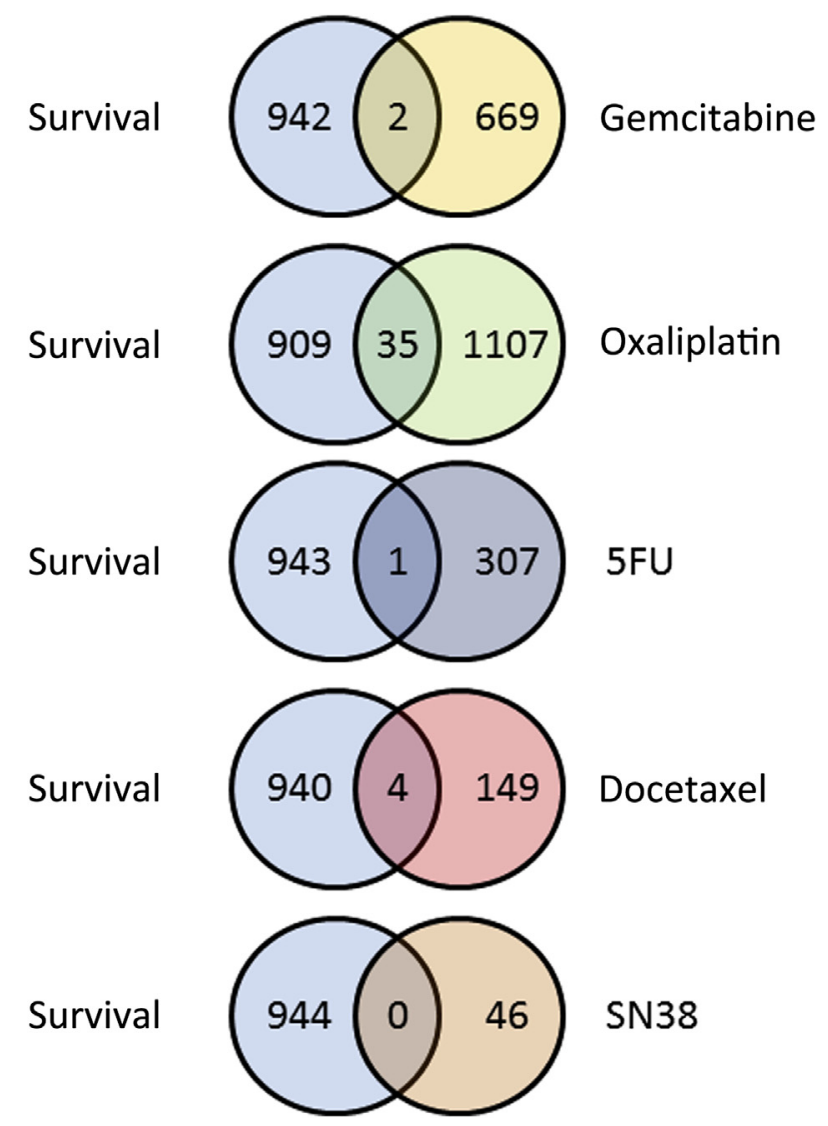

Figure 7 A Venn diagram showing the limited number of genes (in common) associated with resistance or sensitivity to the treatments with those discriminating patients with long- and short-term survival. 5FU, 5-fluorouracil.

Yachida et $\mathrm{al}^{34}$ analyzed the effect of the more frequent somatic genetic alterations-inactivation of CDKN2A, $S M A D 4$, and TP53 and mutation of Kras — on the outcome of patients with a PDAC and found a direct association between accumulation of these genetic abnormalities and short survival. Another important fact to consider concerns data recently reported by Garrido-Laguna et al, ${ }^{35}$ who suggest that xenografts from PDAC tumors grow preferentially when patients have a poor prognosis, indicating a bias for the xenograft approach. From their results, the best-growing xenografts are from patients with inactivation of SMAD4, resistance to gemcitabine, and a gene expression profile enriched in the Notch pathway. This observation seems to make sense but needs to be confirmed by other laboratories. In the present case, of the xenografts studied, only a few showed SMAD4 inactivation (data not shown), sensitivity to gemcitabine was very variable (Figure 5), and genes involved in the Notch pathway showed no particular enrichment (data not shown). However, consistently, aldehyde dehydrogenase activity in xenografts was not determinant in this study because we found $0 \%$ to $34 \%$ positive cells (data not shown).

Another clinically relevant observation concerns the expression profile associated with resistance to some anticancer drugs. Indeed, we observed that 671 genes for gemcitabine,
1107 for oxaliplatin, 308 for 5FU, 149 for docetaxel, and 46 for SN-38 were able to discriminate between tumors sensitive and resistant to each drug. The chemogram we developed includes the most efficient drugs used for treating PDAC patients. Whereas the gene profile associated with gemcitabine, oxaliplatin, and 5FU sensitivity included numerous genes, the number of genes associated with docetaxel and SN-38 was relatively small, suggesting that in the first case the transcriptomic phenotype could be determinant, whereas in the second case this phenotype could be less important. Only a limited number of genes were common between the different drugs, except for oxaliplatin and 5FU (Figure 6B), which is not surprising given that both drugs kill the cell by a related mechanism. ${ }^{36,37}$ Sensitivity to the treatments was patient specific and was not associated with the differentiation degree of the tumor or with the doubling time shown by the primary culture of cancer cells (Figure 4B), which is contrary to expectations, indicating complex molecular mechanisms regulating chemoresistance. Another important observation was that in some cases drugs were unable to kill all the cells, even at concentration as high as $1000 \mu \mathrm{mol} / \mathrm{L}$ (Figure 5), making it impossible to establish their inhibitory concentration of $50 \%$ value. This observation can be explained by the fact that primary cultures are representative of the different cell populations present in the tumor, as heterogeneous as PDAC is known to be. ${ }^{38}$ This could be clinically relevant because the chemogram may detect the percentage of sensitive and resistant cells for a drug and, therefore, may become a helpful tool for the oncologist selecting the second line of treatment for a patient. Other authors have reported a differential sensitivity to anticancer drugs in low-passage pancreatic cancer-derived cells, but no correlation with their RNA profile was reported. ${ }^{39,40}$ However, these authors reported that inactivation of SMAD4 and CDKN2A was associated with resistance to cisplatin and irinotecan and to gemcitabine and mitomycin C, respectively. ${ }^{40}$ Similarly, as for defining whether a PDAC patient will be a short or long survivor, as discussed in Materials and Methods, it would be clinically relevant to determine the expression pattern associated with the sensitivity of the anticancer drugs. In fact, determining the expression of a small number of genes on samples obtained by EUS-FNA could be the first step toward a personalized oncology for PDAC.

Note that the set of genes selected as markers to separate short-term from long-term survival patients were different from genes selected to define sensitivity to the drug (Figure 7). This observation suggests, as expected, that mechanisms of drug resistance are independent of mechanisms defining spontaneous evolution of the disease. We presented data obtained from a relatively small number of samples, but, to our surprise, differences were frequently highly significant, suggesting that this approach is robust. Therefore, we can expect that increasing the number of patients will increase the sensitivity and specificity of data, with a more limited number of genes to be retained to constitute the signature able to determine drug sensitivity and patient outcome. 
In conclusion, we showed that transcriptomic analysis of PDAC xenografts can discriminate short- and long-term survivors and can predict sensitivity to a set of anticancer drugs.

\section{Supplemental Data}

Supplemental material for this article can be found at http://dx.doi.org/10.1016/j.ajpath.2014.11.029.

\section{References}

1. Jemal A, Murray T, Samuels A, Ghafoor A, Ward E, Thun MJ: Cancer statistics, 2003. CA Cancer J Clin 2003, 53:5-26

2. Kindler HL, Ioka T, Richel DJ, Bennouna J, Letourneau R, Okusaka T, Funakoshi A, Furuse J, Park YS, Ohkawa S, Springett GM, Wasan HS, Trask PC, Bycott P, Ricart AD, Kim S, Van Cutsem E: Axitinib plus gemcitabine versus placebo plus gemcitabine in patients with advanced pancreatic adenocarcinoma: a double-blind randomised phase 3 study. Lancet Oncol 2011, 12:256-262

3. Moore MJ, Goldstein D, Hamm J, Figer A, Hecht JR, Gallinger S, Au HJ, Murawa P, Walde D, Wolff RA, Campos D, Lim R, Ding K, Clark G, Voskoglou-Nomikos T, Ptasynski M, Parulekar W; National Cancer Institute of Canada Clinical Trials G: Erlotinib plus gemcitabine compared with gemcitabine alone in patients with advanced pancreatic cancer: a phase III trial of the National Cancer Institute of Canada Clinical Trials Group. J Clin Oncol 2007, 25:1960-1966

4. Van Cutsem E, Vervenne WL, Bennouna J, Humblet Y, Gill S, Van Laethem JL, Verslype C, Scheithauer W, Shang A, Cosaert J, Moore MJ: Phase III trial of bevacizumab in combination with gemcitabine and erlotinib in patients with metastatic pancreatic cancer. J Clin Oncol 2009, 27:2231-2237

5. Costello E, Greenhalf W, Neoptolemos JP: New biomarkers and targets in pancreatic cancer and their application to treatment. Nat Rev Gastroenterol Hepatol 2012, 9:435-444

6. Vincent A, Herman J, Schulick R, Hruban RH, Goggins M: Pancreatic cancer. Lancet 2011, 378:607-620

7. Iovanna J, Mallmann MC, Goncalves A, Turrini O, Dagorn JC: Current knowledge on pancreatic cancer. Front Oncol 2012, 2:6

8. Conroy T, Desseigne F, Ychou M, Bouche O, Guimbaud R, Becouarn Y, Adenis A, Raoul JL, Gourgou-Bourgade S, de la Fouchardiere C, Bennouna J, Bachet JB, Khemissa-Akouz F, PereVerge D, Delbaldo C, Assenat E, Chauffert B, Michel P, MontotoGrillot C, Ducreux M; Groupe Tumeurs Digestives of U, Intergroup P: FOLFIRINOX versus gemcitabine for metastatic pancreatic cancer. N Engl J Med 2011, 364:1817-1825

9. Neesse A, Michl P, Frese KK, Feig C, Cook N, Jacobetz MA, Lolkema MP, Buchholz M, Olive KP, Gress TM, Tuveson DA: Stromal biology and therapy in pancreatic cancer. Gut 2011, 60: 861-868

10. Schreiber FS, Deramaudt TB, Brunner TB, Boretti MI, Gooch KJ, Stoffers DA, Bernhard EJ, Rustgi AK: Successful growth and characterization of mouse pancreatic ductal cells: functional properties of the Ki-RAS(G12V) oncogene. Gastroenterology 2004, 127:250-260

11. Chirgwin JM, Przybyla AE, MacDonald RJ, Rutter WJ: Isolation of biologically active ribonucleic acid from sources enriched in ribonuclease. Biochemistry 1979, 18:5294-5299

12. Irizarry RA, Hobbs B, Collin F, Beazer-Barclay YD, Antonellis KJ, Scherf U, Speed TP: Exploration, normalization, and summaries of high density oligonucleotide array probe level data. Biostatistics 2003, 4:249-264

13. Martinez-Garcia R, Juan D, Rausell A, Munoz M, Banos N, Menendez C, Lopez-Casas PP, Rico D, Valencia A, Hidalgo M: Transcriptional dissection of pancreatic tumors engrafted in mice. Genome Med 2014, 6:27
14. Ishikawa M, Yoshida K, Yamashita Y, Ota J, Takada S, Kisanuki H, Koinuma K, Choi YL, Kaneda R, Iwao T, Tamada K, Sugano K, Mano H: Experimental trial for diagnosis of pancreatic ductal carcinoma based on gene expression profiles of pancreatic ductal cells. Cancer Sci 2005, 96:387-393

15. Buchholz M, Braun M, Heidenblut A, Kestler HA, Kloppel G, Schmiegel W, Hahn SA, Luttges J, Gress TM: Transcriptome analysis of microdissected pancreatic intraepithelial neoplastic lesions. Oncogene 2005, 24:6626-6636

16. Abdollahi A, Schwager C, Kleeff J, Esposito I, Domhan S, Peschke P, Hauser K, Hahnfeldt P, Hlatky L, Debus J, Peters JM, Friess H, Folkman J, Huber PE: Transcriptional network governing the angiogenic switch in human pancreatic cancer. Proc Natl Acad Sci U S A 2007, 104:12890-12895

17. Cavard C, Audebourg A, Letourneur F, Audard V, Beuvon F, Cagnard N, Radenen B, Varlet P, Vacher-Lavenu MC, Perret C, Terris B: Gene expression profiling provides insights into the pathways involved in solid pseudopapillary neoplasm of the pancreas. J Pathol 2009, 218:201-209

18. Abiatari I, DeOliveira T, Kerkadze V, Schwager C, Esposito I, Giese NA, Huber P, Bergman F, Abdollahi A, Friess H, Kleeff J: Consensus transcriptome signature of perineural invasion in pancreatic carcinoma. Mol Cancer Ther 2009, 8:1494-1504

19. Verma G, Bhatia H, Datta M: Gene expression profiling and pathway analysis identify the integrin signaling pathway to be altered by IL1beta in human pancreatic cancer cells: role of JNK. Cancer Lett 2012, 320:86-95

20. Marcotte R, Brown KR, Suarez F, Sayad A, Karamboulas K, Krzyzanowski PM, Sircoulomb F, Medrano M, Fedyshyn Y, Koh JL, van Dyk D, Fedyshyn B, Luhova M, Brito GC, Vizeacoumar FJ, Vizeacoumar FS, Datti A, Kasimer D, Buzina A, Mero P, Misquitta C, Normand J, Haider M, Ketela T, Wrana JL, Rottapel R, Neel BG, Moffat J: Essential gene profiles in breast, pancreatic, and ovarian cancer cells. Cancer Discov 2012, 2:172-189

21. Wang WY, Hsu CC, Wang TY, Li CR, Hou YC, Chu JM, Lee CT, Liu MS, Su JJ, Jian KY, Huang SS, Jiang SS, Shan YS, Lin PW, Shen YY, Lee MT, Chan TS, Chang CC, Chen CH, Chang IS, Lee YL, Chen LT, Tsai KK: A gene expression signature of epithelial tubulogenesis and a role for ASPM in pancreatic tumor progression. Gastroenterology 2013, 145:1110-1120

22. Buchholz M, Kestler HA, Bauer A, Bock W, Rau B, Leder G, Kratzer W, Bommer M, Scarpa A, Schilling MK, Adler G, Hoheisel JD, Gress TM: Specialized DNA arrays for the differentiation of pancreatic tumors. Clin Cancer Res 2005, 11:8048-8054

23. Gress TM, Wallrapp C, Frohme M, Muller-Pillasch F, Lacher U, Friess H, Buchler M, Adler G, Hoheisel JD: Identification of genes with specific expression in pancreatic cancer by cDNA representational difference analysis. Genes Chromosomes Cancer 1997, 19:97-103

24. Crnogorac-Jurcevic T, Efthimiou E, Nielsen T, Loader J, Terris B, Stamp G, Baron A, Scarpa A, Lemoine NR: Expression profiling of microdissected pancreatic adenocarcinomas. Oncogene 2002, 21: 4587-4594

25. Collisson EA, Sadanandam A, Olson P, Gibb WJ, Truitt M, Gu S, Cooc J, Weinkle J, Kim GE, Jakkula L, Feiler HS, Ko AH, Olshen AB, Danenberg KL, Tempero MA, Spellman PT, Hanahan D, Gray JW: Subtypes of pancreatic ductal adenocarcinoma and their differing responses to therapy. Nat Med 2011, 17:500-503

26. Lehmann U, Kreipe H: Real-time PCR analysis of DNA and RNA extracted from formalin-fixed and paraffin-embedded biopsies. Methods 2001, 25:409-418

27. Sohn TA, Yeo CJ, Cameron JL, Koniaris L, Kaushal S, Abrams RA, Sauter PK, Coleman J, Hruban RH, Lillemoe KD: Resected adenocarcinoma of the pancreas-616 patients: results, outcomes, and prognostic indicators. J Gastrointest Surg 2000, 4:567-579

28. Geer RJ, Brennan MF: Prognostic indicators for survival after resection of pancreatic adenocarcinoma. Am J Surg 1993, 165:68-72. discussion-3 
29. You DD, Lee HG, Heo JS, Choi SH, Choi DW: Prognostic factors and adjuvant chemoradiation therapy after pancreaticoduodenectomy for pancreatic adenocarcinoma. J Gastrointest Surg 2009, 13:1699-1706

30. Moon HJ, An JY, Heo JS, Choi SH, Joh JW, Kim YI: Predicting survival after surgical resection for pancreatic ductal adenocarcinoma. Pancreas 2006, 32:37-43

31. Yantiss RK, Chang HK, Farraye FA, Compton CC, Odze RD: Prevalence and prognostic significance of acinar cell differentiation in pancreatic endocrine tumors. Am J Surg Pathol 2002, 26:893-901

32. Wasif N, Ko CY, Farrell J, Wainberg Z, Hines OJ, Reber H, Tomlinson JS: Impact of tumor grade on prognosis in pancreatic cancer: should we include grade in AJCC staging? Ann Surg Oncol 2010, 17:2312-2320

33. Rochefort MM, Ankeny JS, Kadera BE, Donald GW, Isacoff W, Wainberg ZA, Hines OJ, Donahue TR, Reber HA, Tomlinson JS: Impact of tumor grade on pancreatic cancer prognosis: validation of a novel TNMG staging system. Ann Surg Oncol 2013, 20:4322-4329

34. Yachida S, White CM, Naito Y, Zhong Y, Brosnan JA, MacgregorDas AM, Morgan RA, Saunders T, Laheru DA, Herman JM, Hruban RH, Klein AP, Jones S, Velculescu V, Wolfgang CL, Iacobuzio-Donahue CA: Clinical significance of the genetic landscape of pancreatic cancer and implications for identification of potential longterm survivors. Clin Cancer Res 2012, 18:6339-6347

35. Garrido-Laguna I, Uson M, Rajeshkumar NV, Tan AC, de Oliveira E, Karikari C, Villaroel MC, Salomon A, Taylor G, Sharma R,
Hruban RH, Maitra A, Laheru D, Rubio-Viqueira B, Jimeno A, Hidalgo M: Tumor engraftment in nude mice and enrichment in stroma-related gene pathways predict poor survival and resistance to gemcitabine in patients with pancreatic cancer. Clin Cancer Res 2011, $17: 5793-5800$

36. Raymond E, Faivre S, Woynarowski JM, Chaney SG: Oxaliplatin: mechanism of action and antineoplastic activity. Semin Oncol 1998, 25:4-12

37. Thomas DM, Zalcberg JR: 5-fluorouracil: a pharmacological paradigm in the use of cytotoxics. Clin Exp Pharmacol Physiol 1998, 25: 887-895

38. Penchev VR, Rasheed ZA, Maitra A, Matsui W: Heterogeneity and targeting of pancreatic cancer stem cells. Clin Cancer Res 2012, 18 : 4277-4284

39. Kamiyama H, Rauenzahn S, Shim JS, Karikari CA, Feldmann G, Hua L, Kamiyama M, Schuler FW, Lin MT, Beaty RM, Karanam B, Liang H, Mullendore ME, Mo G, Hidalgo M, Jaffee E, Hruban RH, Jinnah HA, Roden RB, Jimeno A, Liu JO, Maitra A, Eshleman JR: Personalized chemotherapy profiling using cancer cell lines from selectable mice. Clin Cancer Res 2013, 19:1139-1146

40. Cui Y, Brosnan JA, Blackford AL, Sur S, Hruban RH, Kinzler KW, Vogelstein B, Maitra A, Diaz LA Jr, Iacobuzio-Donahue CA, Eshleman JR: Genetically defined subsets of human pancreatic cancer show unique in vitro chemosensitivity. Clin Cancer Res 2012, 18: 6519-6530 